\title{
Experiencing Social Augmented Reality in Public Spaces
}

\author{
Anton Nijholt \\ Human Media Interaction, University of Twente, The Netherlands \\ a.nijholt@utwente.nl
}

\begin{abstract}
We present various views on the future use of augmented reality in public spaces. The views address enhanced walking, social activity, appropriation of public spaces, and futuristic social aspects of future outdoor augmented reality. Although we often refer to handheld augmented reality, the focus is on more natural ways of experiencing augmented reality. That is, not using a device at all (projection or mirror-based augmented reality) or using optical seethrough smart eyewear such as smart glasses or lenses that allow the experience of ever-present vision and audio-based augmented reality.
\end{abstract}

\section{CCS CONCEPTS}

- Human-centered computing; • Ubiquitous and mobile computing; Human-centered computing; • Mixed / augmented reality;

\section{KEYWORDS}

Augmented Reality, Public Spaces, Urban Augmented Reality, Social Interaction, Public Events, All-day Augmented Reality, Optical Seethrough, Head-mounted Displays, Smart Glasses, Smart Contact Lenses

\section{ACM Reference Format:}

Anton Nijholt. 2021. Experiencing Social Augmented Reality in Public Spaces. In Adjunct Proceedings of the 2021 ACM International foint Conference on Pervasive and Ubiquitous Computing and Proceedings of the 2021 ACM International Symposium on Wearable Computers (UbiComp-ISWC '21 Adjunct), September 21-26, 2021, Virtual, USA. ACM, New York, NY, USA, 5 pages. https://doi.org/10.1145/3460418.3480157

\section{INTRODUCTION}

In this paper we follow Augmented Reality (AR) pioneer Ronald T. Azuma [1] who predicts that AR, in particular optical see-through glasses, will be the dominant platform and interface, supplanting the smartphone, for accessing digital information. These glasses are seen as "... the best chance of achieving the long-term vision of ubiquitous consumer AR displays." In earlier years Azuma also provided the standard AR definition [2]: AR combines real and virtual objects in a real environment (1), registers (aligns) real and virtual objects (2), and runs interactively, in three dimensions, and in real-time (3). The definition allows various forms of $A R$, such

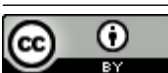

This work is licensed under a Creative Commons Attribution International 4.0 License.

UbiComp-ISWC '21 Adjunct, September 21-26, 2021, Virtual, USA

(c) 2021 Copyright held by the owner/author(s).

ACM ISBN 978-1-4503-8461-2/21/09.

https://doi.org/10.1145/3460418.3480157 as mirror- and projection-based AR, and video and optical seethrough AR (VST and OST AR). These names alone suggest that AR is usually vision-oriented. However, AR applies to all senses and the virtual layer that is superimposed on reality can contain content that together with the real-world content provides visual, auditory, tactile, olfactory, and gustatory experiences or combinations of these experiences, that is, multisensory experiences.

New developments that can be expected are the embedding of AR technology in smart environments and the Internet of Things (IoT), allowing sensors and actuators in the environment, in objects, and a user's (non-AR) wearables to communicate with each other and exchange information. In AR we interactively experience a real-world environment that has been augmented with computergenerated perceptual information. Urban AR that goes beyond navigation support consisting of monochrome textual messages and beyond location-based games is a topic that receives attention. Outdoor experiences are not only multisensorial but also, more than indoors, uncontrolled and spontaneous. Think of the weather, think of traffic, greater freedom of movement, and meeting other people. This leads to other problems and challenges, such as position and orientation handling and limited possibilities to build and use a fully accurate 3D model of the environment that, among other things, would allow proper alignment and handling of occlusion and collision problems.

In this short paper we present some example-based views on issues that emerge in urban AR, that is, in AR-enhanced public space in which we can walk and hang around, meet and interact with other public space users, and attend organized events. AR handhelds, AR glasses and AR contact lenses are the most obvious devices that can be used in such circumstances. In addition, in public spaces there are possibilities for stationary (monitor, kiosk, mirror, screen) AR and spatial AR for which no personal AR device is needed. In our examples we will mainly be concerned with the use of AR glasses but of course, for some virtual content such as sound or smell other sensor-equipped devices or no devices at all are needed.

Presently, both AR glasses as AR contact lenses receive research attention and large companies are investing in research efforts. Facebook has its ARIA project on glasses [3], Apple is on its way to introduce smart glasses and some smaller companies are designing AR contact lenses [4] [5] as the ultimate heads-up display with ultra-thin image and motion sensors and wirelessly connected to a mobile companion app. In this future-oriented paper we assume the presence of all-day wearable AR glasses in the general consumer market. See however [6] [7] [8] for observations on problems with the acceptance of AR glasses in public spaces.

\section{ENHANCED WALKING EXPERIENCE IN AR}

In (optical see-through) AR the world is visible through AR displays. While walking, obstacles can be avoided, physical landmarks are 
visible or even enhanced, and navigation is supported. On the other hand, mainstream AR requires visual attention that can cause distractions and not noticing signboards or pedestrians that stand in the way. This is particularly true for smartphone AR for pedestrian navigation. For smart glasses experiments are needed, as has been done for smartphone AR [9], to study the effect of changes in visual focus on noticing relevant objects during walking.

Standard AR applications provide a live view of the real world overlaid with context-appropriate visual information. They do not necessarily invite social interactions. In theme and amusement parks, the use of AR can stimulate social public interaction. The joint use of location-based exploration apps stimulates the exchange of experiences. Urban (location-based) games that have been designed for smartphones can be enhanced when played with the help of AR eyewear. For various AR applications, such as these urban games, context-appropriate augmentations can be playful and make us laugh together because they are incongruent compared with the usual real-world events and objects.

While walking, we can see virtual graffiti and political messages displayed on or above buildings. For example, WallaMe is an Android app that allows users to hide and share messages in the real world using AR. It was used to display the protest message "Állítsuk meg Orbánt" ("Let’s stop Orbán”) against Hungarian prime-minister Victor Orbán throughout Budapest in 2017, visible through an AR viewer. Apple has launched augmented-reality walks in San Francisco and some other cities. The walks require an app on your iPhone. On these AR enhanced public walking tours virtual art objects integrated into real-world surroundings show up on your iPhone [10]. Similar AR makes it possible to see a giant virtual Donald Trump appear hovering over the Capitol building or, as another example, have the Eiffel Tower neatly integrated into Amsterdam's cityscape.

There are examples where a city's public AR art has been 'vandalized' [11]. When knowing about the GPS coordinates graffiti can be added and virtual changes can be shown. Any public space can be transformed into a space for unauthorized manifestations. While in the past there have been examples of games that included the 'hunting' of surveillance cameras, presently hunting (and hacking) geo-tagged AR sculptures is becoming possible.

Stationary AR can be perceived on billboards, public screens, bus shelters, and street furniture. It is not always recognized as AR when it does not address the sight sense. One example is the piano staircase, well known from Stockholm [12], but also implemented in many other places. Here, the steps of a metro entrance are fitted with sensors and actuators. The sensors detect which step someone steps on, the actuators play a different musical note for each step. This fully complies with the definition of AR as given earlier, as does the Sweden billboard with some sensors that confronts a smoking passer-by with the image and a sound of someone starting coughing, hence, interactive and nicely aligned with reality, or a Paris jaywalker startled violently by the artificially produced sound of squealing tires and a crunching collision. Commercial AR has been displayed on walls of bus shelters, for example, Pepsi Cola's video see-through "Unbelievable Bus Shelter" [13], or on Billboards, for example, Burger King's campaign in Brazil, point your smartphone's camera at a McDonald's billboard advertisement and at your smartphone you see the ad go up in flames [14]. Projectionbased AR in streets and subway passages can be found [15]. In this "Urbanimals" project interactive virtual animals are introduced that are aligned with the real environment, including the human passers-by they interact with.

Virtual humans, or more generally, virtual creatures, have been introduced in AR [16] and we can meet them in outdoor environments. They can represent real humans or animals, in that case we call them avatars, or they can be controlled by algorithms. MARA [17], for example, is a virtual assistant in an outdoor setting, superimposed on the user's view of the real world with an optical see-through head-worn display. Walking or hanging out with a virtual dog is investigated in [18]. If we want a virtual creature in our environment to behave naturally, it should also be aware of changes in the environment, even if they are not caused by a human partner. This can be called multisensorial situational awareness. When we are outside and it starts to rain, our virtual partner should become wet too. In [19] similar examples of external stimuli that should prompt multisensorial startling behavior are mentioned.

We can compare walking in AR with walking in VR and see what problems are encountered there. In fully immersed VR we do not perceive the real world around us. Nevertheless, nowadays, standalone VR headsets also allow walking. Artificial locomotion techniques for small physical spaces can be replaced by a more natural way of walking around in room-scale or larger indoor and outdoor spaces by having location-based VR setups. Artificial walking in VR includes walking-in-space, treadmill, and redirectional walking. When walking is not an automatic process anymore it has consequences for issues such as maintaining a stable position and, more general, effects on presence. From experiments with standalone VR headsets and 'natural' walking in sports-arena-sized virtual environments, it has been suggested that walking-based VR in wide-area environments can become feasible for general audiences [20]. In such situations safety of VR users is of course the first priority. AR users can also end up in unsafe situations, as has been shown with Pokémon Go (although with handheld AR instead of head-worn AR) and perhaps with AR when the reality is obscured by too many virtual additions and changes (immersive AR). Issues such as presence and maintaining a stable position can emerge then as well.

An interesting, VR approach to a walking experience is Microsoft's DreamWalker project [21]. Although it is not AR, several research aspects of this project can be investigated in immersive AR as well. In DreamWalker a user with a VR headset walks from one place to another in the real world, while experiencing a completely different urban environment. The path in the virtual environment is aligned with the real-world path, obstacles discovered along the way in the real world (not seen by the VR user but by cameras mounted on the VR device), for example, pets or other pedestrians, lead to real-time corrections of the virtual path that is presented to the user. The aim is that users can safely take their daily walk to work while experiencing a city of their choosing. Possible downsides of the pursuit of more immersion in AR are unwanted emotional and behavioral changes when leaving the AR world. 


\section{SOCIAL AR ACTIVITY IN PUBLIC SPACES}

Smartphone AR, in particular AR games, can enhance social interaction between people, not only in the game but also in reality. For example, Pokémon Go users enjoy getting together and sharing experiences. In other urban games, meeting, competing, or cooperating with others is part of the game. This will not be different if we do not play such games with a (video see-through) smartphone, but with optical see-through glasses. In public entertainment activities, participants will not use AR in isolation, there will be physically co-located AR activities. Projection-based AR, whether it is a projection on an interior table, an arrangement of physical objects, or a complete building, is of course ideally suited for social interaction, not only between players but also with and between audience members.

AR technology can be used by multiple users sharing the same physical space and interacting with shared virtual content. Creative and mischievous use of AR technology includes hacking, for example by unauthorized view sharing and disrupting views by making changes in the properties of the virtual content that appears in the virtual layer and is connected with the real world. Besides, as discussed in [22], especially multi-application AR systems are at risk of blocking or obscuring important real-world content (e.g., stairs, a person, or an oncoming vehicle) and displaying distracting visual AR. The safety and security implications of an unregulated push of virtual content in public and commercial spaces need to be investigated and require design policies for AR platforms [22].

Social interaction is not limited to face-to-face interaction or interaction with someone remotely. Several people can be involved in an interaction, in home situations as well as in public spaces, and interactions can be completely non-verbal. With the help of AR technology, humorous and playful events can be created in public spaces. Such events are inextricably linked to social interactions. They draw audiences and cause audience interaction. Organized spatial AR events, for example, the integration of play elements or interactive art with the architecture of buildings or other physical surfaces such as billboards, bus shelters, or big screens in station halls, attract both actively and passively engaged audiences that shape the (social) event. Playful augmented reality has been made part of public spaces, inviting passers-by to become active or have them enjoy seeing other people become active [23] [24] [15]. Mirror $\mathrm{AR}$ is a type of video-supported augmented reality where the camera is aimed at the user and the user's image, possibly manipulated, is projected as an avatar in a computer-generated world displayed on a screen or building which allows entertaining interactions with the user. Since this augmented world is visible to passers-by and bystanders, they may feel invited to become participants too [25] The gestures and movements of participants detected by cameras also allow joint activity with virtual creatures that are projected into such a world.

\section{AR APPROPRIATION OF PUBLIC SPACES}

In [3] Facebook's approach to the modeling of 'reality is explained as part of the ARIA project. Reality is modeled in Live Maps, a 3D map of the world that can be shared with others or their avatars and that tells you about your precise location and orientation and how your augmented world looks from your first-person point of view. Facebook researchers aim at introducing this platform with a bottom layer that provides the AR user with locations, built on it is a $3 \mathrm{D}$ map of static structures (physical surfaces, structures, and objects), additional layers of movable objects and their properties, and a layer that contains virtual objects and their alignments with the real world as it is represented in the other layers. Relationships, histories, predictions for entities and events are meant to be stored in these layers in an egocentric way. While surveying the environment, a user helps to model the augmented urban environment from a first-person point of view.

This approach, or rather its comprehensiveness, led to criticism. For example, Jessica Conditt [26] mentions "All of this will eventually coalesce into a new, Facebook-controlled reality, laid directly on top of the physical world. ... It's also deeply unsettling. In this vision of the future, Facebook is literally building a new reality one that it can change on a whim, removing objects and mechanics that don't mesh with its corporate ideals and adding objectives that have been purchased by the highest bidder. ... If AR works the way Facebook envisions, we'll use it to navigate our daily lives and turn to it at every step for information, connection, entertainment, and employment. ... With the emergence of mainstream AR, our reality will be distorted by a for-profit organization, guaranteed."

This author is not alone in her criticism. We have seen the privatization of physical public spaces where citizens must submit to camera-enforced consumer behavior, wavelength auctions that allow commercial exploitation of the air, and now we can observe the augmented reality exploitation of public spaces. The right to mark up the city is not reserved for a municipality's public works. Billboards and large screens already define the cityscape. Miguel Sicart [27] mentions that advertisement and commercial enterprises are expected to pay for the use of public spaces. There are regulations about the use of public space. However, AR interfaces circumvent these regulations. Urban games such as Pokémon GO can be exploited for commercial interests and public spaces can be instrumentalized for private profit. "AR can erode the use of public spaces." [27]. Indeed, there are examples of companies struggling with how to acquire ownership of their customer reach and how to compete with their competitors in the augmented reality public spaces. This AR appropriation of public spaces can be illustrated with an example from China. In 2013 the Chinese E-commerce grocer Yihaodian [28] opened many virtual 3D stores, virtually located at some of China's iconic landmark locations as well as directly in front of their competitor supermarket stores. In Burger King's advertising campaign (section 2) the competitor's advertisements are appropriated with smartphone AR technology. After the flames have burned away, your screen notifies you that you can get a free burger from a nearby Burger King restaurant [14].

\section{FUTURE AND FUTURISTIC AR IN PUBLIC SPACES}

A few observations on future social AR as it is foreseen in movies and promotional videos are in order. We can expect that real-time detected and collected publicly or commercially available personality characteristics, moods, and emotions will play a role in future AR-supported social interactions made possible by glasses and lenses. 
An interesting example of AI-guided alignment of virtual content superimposed on the real world, although only given a fake implementation, can be found in a "Daily Show" broadcast of talk-show host Jon Stewart [29]. It is a detector that recognizes the humor in a conversation and prompts its user to start laughing. In his TV show, it was humorously suggested that the detector was used by Hillary Clinton, allowing her to laugh at the right time during interviews, whether she recognized the humorous comment herself or was triggered by the system. In this example, the virtual content was provided auditory but obviously, the message "humorous remark detected" could have been displayed on smart glasses or lenses instead, making it more recognizable as regular AR.

We can see more examples of future AR profiling in movies and television series. Sometimes they are being overtaken by reality, and the only futuristic aspect is that rather than AR glasses, AR contact lenses or retina implants are used. In the 2012 short futuristic film "Sight" [30] a dating app is introduced that provides information about a partner through the use of facial recognition and during the conversation, based on the app's interpretation of verbal and nonverbal behavior, suggestions are made about how to continue the conversation, such as change the subject, try a smile, or consider to wrap up. In "Nosedive", the 2016 TV Episode of "Black Mirror" [31], you get rated by everyone you meet and this rating is also shown to everyone you meet. People adjust their behavior to their rating and that of others. When your behavior does not comply with the rules it is also possible to get a penalty and decrease of rating from the authorities. As a consequence, there will be restrictions on traveling by plane or using a private car. In [32] we discussed China's social credit system that has similar aims. In the 2018 Canadian movie "Anon" we also see information about the persons we meet on the street or with whom we are communicating displayed on our AR contact glasses [33]. Both in the "Sight" and the "Nosedive" movie behavioral and affective information in a social setting is measured and plays a role in the affective interaction between AR users.

The way AR is featured in movies and TV series is fed by companies' promotional videos. Looking back, the 2013 promotional videos of Google Glass and, as another example, the company Infinity AR, caused outrage on privacy invasion issues and some of the applications that were shown; for example, what was called sociopathic behavior of the glass user, such as getting help in flirting and setting up dates. As mentioned in [34] "It's a sort of bachelor fantasy in which a man gets AR help from his smart glasses to pick his wardrobe, drive his Ferrari to an upscale pool hall, get advice on his pool shooting, and impress the attractive bartender with the knowledge that she's a Gemini." On the other hand, the AR technology that was really shown by companies was in fact rather modest: glasses with only a small screen for Internet search results or video connections, the information in text or picture form about realworld objects and people who come into view, recommendations, and services from third-party companies, et cetera. Interestingly, in the promotional videos and the AR Sci-Fi series hardly or no references can be found to the ability to augment the real-world scenes with aligned 3D objects and virtual humans, or to make real-world objects invisible in real-time in diminished reality. In the "Black Mirror" episode "White Christmas" (2014) remote guidance for flirting is provided by a human assistant instead of an A.I. and rather than using a form of diminished reality to make people invisible they are 'blocked' by giving them a ghost-like appearance.

\section{CONCLUSIONS}

In this nontechnical vision-oriented paper we surveyed various issues that are related to the employment of AR technology in urban environments. The underlying assumption is that we will inhabit an ever-present AR world that is displayed on our all-day wearable $\mathrm{AR}$ glasses or lenses. Smartness is provided by the integration of AR devices with our other wearables, the IoT, and A.I. In the various sections this was illustrated from four viewpoints: walking in AR, social activity in AR, ownership of AR, and AR as it is profiled in movies and TV series.

At the moment we have to be content with optical see-through AR devices such as the Microsoft's Hololens and the Magic Leap OST AR devices. A very recent survey on optical see-through AR HMDs can be found in [35]. A video see-through feature (Passthrough) has recently been introduced for Facebook's Oculus Quest wireless VR HMD, where its tracking cameras provide the user with a view on their real-world surroundings. Presently, for public spaces and urban environments we see AR applications appear that go beyond navigation and annotation, such as in (landscape) architecture, urban planning, localized games, cultural heritage and tourism, guides and social companions, and entertainment. Others have to be found.

Although the various subjects have only been treated lightly, sometimes by just giving examples, we can conclude that the integration mentioned above receives little or no attention. AR is usually still treated as an isolated technology with vision as the dominant sense. Virtual content should be interpreted more broadly and the associated 'alignment' with the real world should also relate to (social) behaviors, activities, and (real-world) events that are not necessarily under the control of the user or digital devices. This broader interpretation of alignment needs to be driven by dynamic information obtained from ubiquitous sensors and actuators that must be interpreted with the help of A.I.

Interestingly, in Sci-Fi movies and TV series about AR-enhanced worlds, AR technology is often limited to providing textual information to the user about his environment or interaction with human partners and floating in the air before the real-world scene. Application of AI becomes visible when advising on how to continue an activity or interaction such as in "Sight". But by no means are augmented worlds created with their residents equipped with AR contact lenses, such as we know from Vernor Vinge's Rainbow Ends [36] [37], where users can redress the environment to their taste.

We are aware that each of the topics here can be explored indepth in future articles that do much more justice to research already done and published. We hope to have provided an impetus for this.

\section{REFERENCES}

[1] R.T. Azuma, 2019. The road to ubiquitous consumer augmented reality systems. Hum Behavior \& Emerging Technologies 1, 26-32.

[2] R.T. Azuma, 1997. A Survey of Augmented Reality. Presence: Teleoperators and Virtual Environments 6:4, 355-385.

[3] M. Abrash, 2020. Project ARIA. In: Facebook Connect Keynote: Oculus Quest 2, Project Aria \& More! $1.33 .20-1.47 .50$. https://www.youtube.com $/$ watch?v= woXmJMw2lTM 
[4] Samsung Electronics Co., Ltd., 2015. Smart contact lenses for augmented reality and methods of manufacturing and operating the same. US 2016/0091737 A1, (Granted in 2019).

[5] T.S. Perry, 2020. Augmented Reality in a Contact Lens: It's the Real Deal. IEEE Spectrum. [Online, 16 January 2020] https://spectrum.ieee.org/view-from-thevalley/consumer-electronics/portable-devices/ar-in-a-contact-lens-its-thereal-deal

[6] B. Due, 2015. The social construction of a Glasshole: Google Glass and multiactivity in social interaction. PsychNology Journal, 13(2-3), 149-178.

[7] P.A. Rauschnabel, D.W.E. Hein, J. He, Y.K. Ro, S. Rawashdeh, and B. Krulikowski, 2016. Fashion or Technology? A Fashnology Perspective on the Perception and Adoption of Augmented Reality Smart Glasses. i-Com, 15(2), 179-194.

[8] S. Kernaghan 2016. Google Glass: An Evaluation of Social Acceptance. Thesis, School of Engineering and Digital Arts, University of Kent.

[9] S. Yoshiki, H. Tatsumi, K. Tsutsumi, Y. Okumura, and M. Otsuka, 2019. Effects on space recognition of walking through augmented reality. Urban and Regional Planning Review, 6, 84-95.

[10] P. Rubin, 2019. Apple puts the AR in 'Art'. Wired. https://www.wired.com/story/ apple-ar-art-walk/

[11] C. Voon, 2017. Artist Vandalizes Virtual Koons Sculpture, Questioning Silicon Valley's Fake Public Space. October 5, 2017. https://hyperallergic.com/404143/ sebastian-errazuriz-jeff-koons- snapchat/

[12] A. Nijholt, 2017. Towards Playful and Playable Cities. Chapter 1 in: A. Nijholt (ed.) Playable Cities. Gaming Media and Social Effects. Springer, Singapore, 1-20.

[13] Grand Visual, 2014. Unbelievable Bus Shelter. https://grandvisual.com/work/ pepsi-max-bus-shelter/

[14] Burger King, 2017. https://boundtowow.com/burn-that-ad-burger-king/

[15] S. Dobiesz and A. Grajper, 2016. Animating the Static. Case Study of The Project "Urbanimals" Enhancing play in the cities through an augmented and interactive environment. Complexity \& Simplicity. 34th International Conference on Education and Research in Computer Aided Architectural Design in Europe (eCAADe 34), Volume 1. A. Herneoja, T. Österlund, and P. Markkanen (Eds). Oulu School of Architecture, Oulu, Finland, 691-697.

[16] A. Nijholt, 2021. Augmented Reality Humans: Towards Multisensorial Awareness. 6th International Conference on Digital Economy (ICDEc 2021), R. Jallouli, M.A.B. Tobji, H. Mcheick, G. Piho (Eds.), Lecture Notes in Business Information Processing. Springer, Cham, Switzerland, to appear.

[17] A. Schmeil and W. Broll, 2007. MARA - A Mobile Augmented Reality-Based Virtual Assistant. 2007 IEEE Virtual Reality Conference (Charlotte, NC, USA) IEEE, New York, USA, 267-270.

[18] N. Norouzi, K. Kim, M. Lee, R. Schubert, A. Erickson, J.N. Bailenson, G. Bruder, and G. Welch, 2019. Walking Your Virtual Dog: Analysis of Awareness and Proxemics with Simulated Support Animals in Augmented Reality. IEEE International Symposium on Mixed and Augmented Reality (ISMAR), 253-264.

[19] H. Schraffenberger and E. van der Heide, 2014. Everything augmented: On the real in augmented reality. Journal of Science and Technology of the Arts, 6(1), $17-29$.
[20] E. Sayyad, M. Sra, and T. Höllerer, 2020. Walking and Teleportation in Widearea Virtual Reality Experiences. IEEE International Symposium on Mixed and Augmented Reality (ISMAR), Recife/Porto de Galinhas, 608-617.

[21] J. Yang, C. Holz, E. Ofek, and A.D. Wilson, 2019. DreamWalker: Substituting RealWorld Walking Experiences with a Virtual Reality. 32nd Annual ACM Symposium on User Interface Software and Technology (UIST '19). ACM, New York, USA, 1093-1107.

[22] K. Lebeck, K. Ruth, T. Kohno, and F. Roesner, 2017. Securing Augmented Reality Output. IEEE Symposium on Security and Privacy (SP), San Jose, CA, 320-337.

[23] A. Nijholt, 2020. Playful and Humorous Interactions in Urban Environments Made Possible with Augmented Reality Technology. Interactivity, Game Creation, Design, Learning, and Innovation. ArtsIT 2019, A. Brooks and E. Brooks (Eds.), LNICST, vol 328. Springer, Cham, Switzerland, 273-289.

[24] A. Nijholt, 2021. Humorous and Playful Social Interactions in Augmented Reality. Contribuciones a la Lingüística y a la Comunicación Social. Tributo a Vitelio Ruiz Hernández. Sixteenth International Symposium on Social Communication. Santiago de Cuba, Cuba, 167-173.

[25] P. Dalsgaard and K. Halskov, 2010. Designing urban media façades: cases and challenges. SIGCHI Conference on Human Factors in Computing Systems (CHI '10). ACM, New York, USA, 2277-2286.

[26] J. Conditt, 2019. If Facebook owns reality, it'll be impossible to opt out. How much data are you willing to sacrifice for a more comfortable world? https://www.engadget.com/2019-09-25-facebook-ar-livemaps-futurereality-oculus-connect-6.html, September 25, 2019.

[27] M. Sicart, 2017. Reality has always been augmented: Play and the promises of Pokémon GO. Mobile Media \& Communication, 5(1), 30-33.

[28] Yihaodian, 2021. Chinese E-commerce Grocer Yihaodian \& O\&M Advertising Shanghai's 1,000 Virtual Stores App Case Study. https://youtu.be/hJqIpIlR3nI, 2013. Retrieved 14 February 2021

[29] J. Stewart, 2007. The Daily Show with Jon Stewart: Hillary's Laugh Track, Season 12 E 120, 09/25/2007. https://www.cc.com/video/1xwx9x/the-daily-show-withjon-stewart-hillary-s-laugh-track

[30] D. Lazo and E. May-Raz, 2012. Sight. Retrieved January 29, 2021, from https: //vimeo.com/46304267. See also https://www.sightextended.com/

[31] J. Wright, 2016. Black Mirror Series 3 Episode 1: Nosedive.

[32] A. Nijholt, 2020. City Residents as Videogame Characters in Smart Urban Environments. In: A. Nijholt (Ed.) Making Smart Cities More Playable. Gaming Media and Social Effects. Springer, Singapore, 355-377.

[33] A. Niccol, 2018. Anon. Canada, https://www.imdb.com/title/tt5397194/

[34] S. Shankland, 2014. Infinity AR: We'll fulfill sci-fi promise of augmented reality. https://www.cnet.com/news/infinity-ar-well-fulfill-sci-fi-promise-ofaugmented-reality/

[35] Y. Itoh, T. Langlotz, J. Sutton, and A. Plopski. 2021. Towards Indistinguishable Augmented Reality: A Survey on Optical See-through Head-mounted Displays. ACM Comput. Surv. 54, 6, Article 120 (July 2021), 36 pages.

[36] V. Vinge, 2004. Synthetic serendipity. IEEE Spectrum, vol. 41, no. 7, 35-44.

[37] V. Vinge, 2006. Rainbows End. Tor Books, New York, USA. 